\title{
Lossless Image Compression using Adaptive Predictive Coding of Selected Seed Values
}

\author{
Ghadah Al-Khafaji, PhD \\ Dept. of Computer Science, \\ Baghdad University, \\ College of Science.
}

\author{
Haider Al-Mahmood \\ Dept. of Computer Science, \\ Al-Mustansiriya University, \\ College of Science.
}

\begin{abstract}
In this paper, two proposed effective selection seed values techniques adopts to enhance the predictive coding efficiency.
\end{abstract}

\section{Keywords}

Data compression, redundancy, lossless, seed values and predictive coding

\section{INTRODUCTION}

In general, the multimedia field highly relied on data compression that implies image, audio and video. Thus, today data compressions become intensively research area that continuously constructs standard compression system like JPEG, MPEG, MP and H.265.

Basically, the data compression based on removing or eliminating the redundancy(s) as much as possible. In image case, the redundancy can be categorized into statistical redundancy and psychovisual redundancy, for the former this encompass the lossless image compression, while for the latter only or with the former this encompass the lossy image compression, for more details see [1-8]

The predictive coding one of the well known image techniques still under the development that utilized the spatial domain efficiently that involving two steps of prediction (i.e., creating an approximate image to the original one) and differentiation (i.e., finding the residual between the original and the prediction image) [9]. The modelling concept is the core of the predictive coding that simply means formulate the embedded image redundancy (i.e., spatial redundancy) mathematically using the deterministic part (that required definition of the model order, model dependency form and model structure) and probabilistic part [9-16].

The predictive coding originally suffers from the seed value that corresponds to the initial modelling coding to construct the predicted image, that unfortunately reduced the compression efficiency of the technique [9,17-19].

In this paper, an efficient lossless image compression technique is adopted that effectively overcome the seed value problem that discussed in details in section 2, also the results and discussion illustrated in section 3 .

\section{PREDICTIVE CODING}

The predictive coding techniques essentially use the seed values to create the predicted image where the spatial domain of the embedded correlation neighbours exploited. Two algorithms adopted to use the seed values efficiently/effectively for lossless image compression.

\subsection{Seed Difference}

The first technique based on partitioning the square input image $(\mathrm{NxN})$ (uncompressed image) into fixed square block sizes (nxn), each segment lock utilized only the left and bottom seed values (i.e., initial condition does not include all the first row and column seed values but only selected values from them) to create the predicted values of the current pixel and the left bottom pixel based on the horizontal and vertical differences according to equations below (1-6), figure (1) shows the idea clearly.

$$
\begin{aligned}
& \text { HorizontalX Predcurr }=\text { Xvalue }- \text { Left. } \\
& \text { VerticalX Predcurr }=\text { Xvalue }- \text { Bottom } \\
& X \text { Predcurr }=\left\{\begin{array}{l}
\text { HorizontalX Predcurrif (HorizontalX } \operatorname{Pr} \text { edcurr }<=\text { VerticalX Predcurr) } \\
\text { VerticalX Predcurrelse }
\end{array}\right.
\end{aligned}
$$

HorizontalLeftBottom Predcurr $=$ LeftBottom - Bottom (5)

VerticalLeftBottom Predcurr $=$ LeftBottom - Left

LeftBottom Predcurr $=\left\{\begin{array}{l}\text { HorizontalLeftBottom Predcurrif (HorizontalLeftBottom Predcurr }<=\text { VerticalLeftBottom Predcurr) } \\ \text { VerticalLeftBottom Predcurrelse }\end{array}\right.$

The predicted image depends on the lowest difference values of either horizontal or vertical difference values of the current pixel value and the left bottom value.
Lastly, the residual (residue) as the difference can be computed as a difference between the original image and the predicted one, reversely to reconstruct the image back adds the residual to the predicted image. 


\begin{tabular}{|l|l|}
\hline Left Bottom & Bottom \\
\hline Left & Xvalue \\
\hline
\end{tabular}

\begin{tabular}{|l|l|}
\hline Left Bottom Predictedvalue & Bottom known pixel value \\
\hline Left known pixel value & XPredictedvalue \\
\hline
\end{tabular}

\begin{tabular}{|l|l|}
\hline 120 & 119 \\
\hline 120 & 114 \\
\hline
\end{tabular}

HorizontalX Predcurr $=6$

VerticalX Predcurr $=5$

$X$ Predcurr $=119$
HorizontalLeftBottom Predcurr $=0$

VerticalLefiBottom Predcurr $=1$

LeftBottom Predcurr $=120$

\begin{tabular}{|l|l|}
\hline 120 & 119 \\
\hline 120 & 119 \\
\hline
\end{tabular}

\subsection{Seed of Difference and Minimum Value}

The second technique simply also starts from the original square image $(\mathrm{NxN})$ that partitioned into the fixed square block of sizes (nxn), each segment lock utilized only the left and bottom seed values, the left bottom pixels predicted according to equations (4-6), while for the predicted values of the current pixel the horizontal and vertical differences calculated as in equations (1-2), if one of the differences is

equal to zero then either the horizontal or vertical difference is applied, otherwise the lowest difference values of either horizontal or vertical difference values subtracted from the selected difference value of minimum difference value according equations(7), as illustrated in figure (2).

$X$ Predcurr $=\left\{\begin{array}{l}\text { HorizontalX Predcurr }- \text { Bottomif }(\text { HorizontalX Predcurr }<=\text { VerticalX Predcurr }) \\ \text { VerticalX Predcurre }- \text { Leftlse }\end{array}\right.$

\begin{tabular}{|l|l|}
\hline Left Bottom & Bottom \\
\hline Left & Xvalue \\
\hline
\end{tabular}

\begin{tabular}{|l|l|}
\hline Left Bottom Predictedvalue & Bottom known pixel value \\
\hline Left known pixel value & XPredictedvalue \\
\hline
\end{tabular}

\section{Original image block}

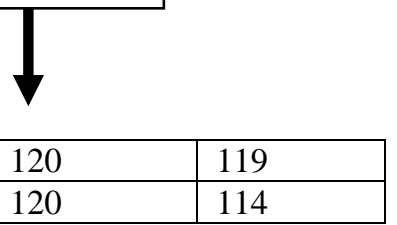

HorizontalX Predcurr $=6$

VerticalX $\operatorname{Pr}$ edcurr $=5$

$X$ Predcurr $=119-5$

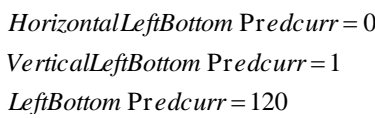

LeftBottom Predcurr $=120$

Fig. (2): Seed difference and minimum technique

Also the reconstructed image based on the predicted along with the residual images.

\section{EXPERIMENTAL AND RESULTS}

Lossless techniques efficiency based on quantitatively measuring of compression performance only, the compression ratio (which is the ratio of the original image size to the compressed size) is generally adopted; also here six tested standard square gray scale images selected as shown in fig (3) that implies of natural and medical images.

\section{Predicted image block}

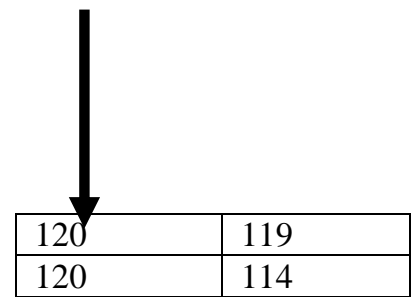




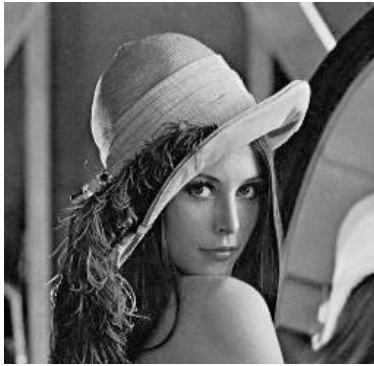

Lena

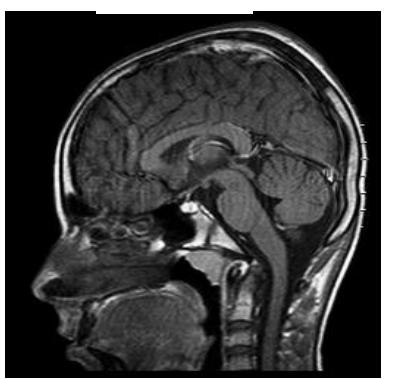

Brain (MR)

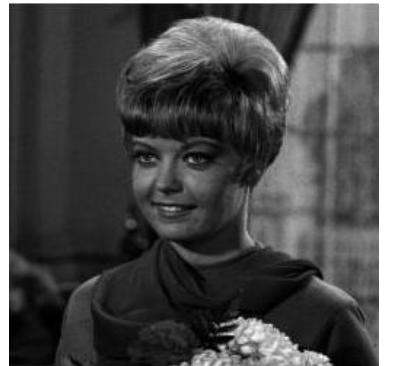

Girl

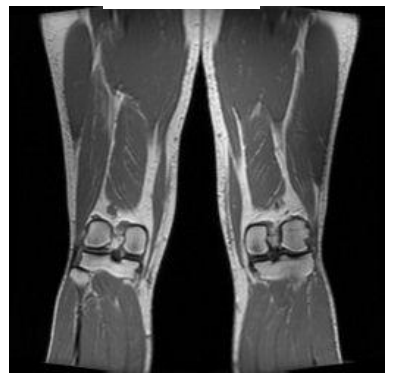

Knee (MR)

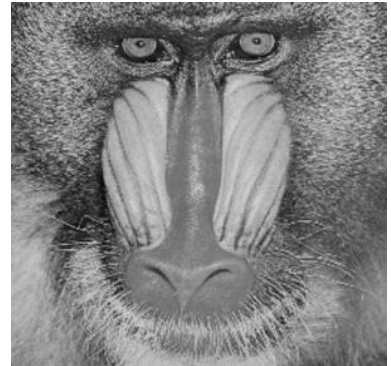

Baboon

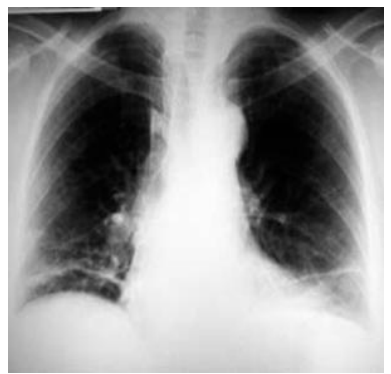

Chest (X-ray)

Fig. (3): Tested images gray scale images of size $256 \times 256$.

The results shown in table (1)illustrates clearly the superior performance of the second suggested techniques of Seed of Difference and Minimum Value compared to the first technique of Seed Difference, due to high prediction image quality that resemble the original image leading to small prediction error (residual). In other words, the compression ratio directly affected by the way of selected the seed values, since less residual error required efficient selected seed values.

Lastly, the results also showed that there is a trade-off between the technique efficiency or the compression performance (i.e., compression ratio) and the image structure (image details and variation), where high compression ratio of medical images of small image details and low variation compared to natural images of high details and large variation.

Table 1: Compression ratio of the two proposed techniques of the tested images using $(2 \times 2)$ block sizes.

\begin{tabular}{|l|l|l|}
\hline \multirow{2}{*}{$\begin{array}{l}\text { Tested } \\
\text { Image }\end{array}$} & Seed Difference & $\begin{array}{l}\text { Seed of Difference and } \\
\text { Minimum Value }\end{array}$ \\
\cline { 2 - 3 } & $\begin{array}{l}\text { Compression } \\
\text { Ratio }\end{array}$ & Compression Ratio \\
\hline Lena & 7.5642 & 9.7263 \\
\hline Girl & 8.8431 & 11.2740 \\
\hline Baboon & 6.6406 & 8.3977 \\
\hline $\begin{array}{l}\text { Brain } \\
\text { (MR) }\end{array}$ & 9.1800 & 11.4674 \\
\hline $\begin{array}{l}\text { Knee } \\
(\text { MR) }\end{array}$ & 10.1954 & 13.9201 \\
\hline $\begin{array}{l}\text { Chest }(\mathrm{X}- \\
\text { ray) }\end{array}$ & 11.2142 & 13.1546 \\
\hline
\end{tabular}

\section{REFERENCES}

[1] Ilam Parithi, T. and Balasubramanian, R. 2015. A Review on Different Lossless Image Compression Techniques. International Journal of Modern Sciences and Engineering Technology, 2(4), 86-94.
[2] Malwinder, K. and Navdeep, K. 2015. A Literature Survey on Lossless Image Compression. International Journal of Advanced Research in Computer and Communication Engineering, 4(3) 491-493.

[3] Kumari, A. and Priya, D. 2015. A Survey on Lossless Compression Techniques. Proceedings of 13th International Conference, Bengaluru, India, 20-27.

[4] Shilpa, I. and Deepak K. 2014. A Review on - Lossless Image Compression Techniques and Algorithms. International Journal of Computing and Technology, 1(9), 457-460.

[5] Amruta, S. and Sanjay L. 2013. A Review on Lossy to Lossless Image Coding. International Journal of Computer Applications, 67(17), 9-16.

[6] Venkatraman, B., Vaithiyanathan, V. and Karthikeyan, B. 2013. Review on Lossless Image Compression Techniques for Welding Radiographic Images. Research Journal of Applied Sciences, Engineering and Technology 5(1), 167-170.

[7] Ferni Ukrit, M., Umamageswari, A. and Suresh, G.R. 2011. A Survey on Lossless Compression for Medical Images. International Journal of Computer Applications, 31(8), 47-50.

[8] Sayood, K. Introduction to Data Compression. 2006. 3rd edn.Elsevier Inc., San Francisco United States of America

[9] Al-Khafaji, G. 2012. Intra and Inter Frame Compression for Video Streaming. Ph.D. thesis, Exeter University, UK.

[10] Al-Mahmood, H. and Al-Rubaye. Z. 2014.Lossless Image Compression Based on Predictive Coding and Bit Plane Slicing. International Journal of Computer Applications, 93(1)1-6.

[11] Sinan, Th. 2014. Medical Image Compression. Diploma, Dissertation, Baghdad University, Collage of Science 
[12] Ghadah, Al-K. 2013. Hierarchal Autoregressive for Image Compression. The Proceeding of the 4th Conference of College of Education for Pure Science, Thi-Qar University, 4(1), 235-241.

[13] Gray, R. M. 2010. A Survey of Linear Predictive Coding: Part I of Linear Predictive Coding and the Internet Protocol. Foundations and Trends in Signal Processing, 3(3), 153-202.

[14] Fletcher, A.K. 2007. Robust Predictive Quantization: Analysis and Design Via Convex Optimization. Signal Processing, 1(4), $618-632$.

[15] Chen, Y-T. and Tseng, D-C. 2007. Wavelet-Based Medical Image Compression with Adaptive Prediction. Computerized Medical Imaging and Graphics, 31, 1-8.
[16] Burgett, S. and Das, M. 1993. Predictive Image Coding using Multiresolution Multiplicative Autoregressive Models. Proceedings of the IEEE, 140(2), 127-134.

[17] 17-Murakami, H., Matsumoto, S., Hatori, Y. and Yamamoto, H. 1987. 15/30Mbit/s Universal Digital TV Codec Using a Median Adaptive Predictive Coding Methods. IEEE Transactions on Communications, 35(6), 637-645.

[18] Salo, J. and Neuvo, Y. 1988. A New Two Dimensional Predictor Design for DPCM Coding of Video Signals. Signal Processing of HDTV, 207-211.

[19] Perng, M. H. and Lin, H. H. 2006. Image Compression in the Wavelet Domain Using an AR Texture Model with Compressed Initial Conditions. Proceedings of the 5th WSEAS International Conference on Signal Processing, Robotics and Automation, 46-51. 